ПРИЛОЗИ, Одделение за природно-математички и биотехнички науки, МАНУ, том 38, бр. 2, стр. 147-152 (2017) CONTRIBUTIONS, Section of Natural, Mathematical and Biotechnical Sciences, MASA, Vol. 38, No. 2, pp. 147-152 (2017)

\title{
CURVATURE AND GROUP THEORY
}

\author{
Ross Geoghegan \\ Department of Mathematics, Binghamton University, Binghamton, USA \\ e-mail: ross@math.binghamton.edu
}

\section{INTRODUCTION}

This informal article covers the same material as was in my talk to the Macedonian Academy of Sciences and Arts in May 2016 on the occasion of my becoming a foreign member of that academy. I am honored by being elected to this, and I thank my Macedonian hosts, particularly my former doctoral student and longtime friend Acadamician Donco Dimovski.

It was suggested that the talk should be of a rather general nature, and that the write-up should be readable by students. So what follows should be regarded as an invitation to go deeper, not a full expository paper. A general reference for the material on $C A T(0)$ geometry is [1]. All the other material discussed here is dealt with in full detail in my book [5], where original sources are also given.

The goal of the talk is to describe some equivalences between algebraic and topological statements - statements which superfically look entirely different. Most of these equivalences are known theorems, though the last one - the most intriguing - involves an open question.

While I do things here in the elegant world of $C A T(0)$ geometry, I should say that some of the statements can be generalized to arbitrary finitely presented groups, though in that generality they are not as pretty.

\section{CAT(0) GEOMETRY}

Throughout this article $M$ denotes a proper $C A T(0)$ metric space. More precisely, this means

- $M$ is a metric space, with metric $d$;

- $M$ is proper, i.e. closed balls are compact;

- $M$ is a geodesic space, i.e. for any two points $x$ and $y$ there is an isometric embedding of the closed interval $[0, d(x, y)]$ into $M$ with 0 going to $x$ and the number $d(x, y)$ going to $y$. Such a path is called a geodesic.

- $M$ satisfies the $C A T(0)$ comparison axiom, which I will now explain.

The $C A T(0)$ axiom compares geodesic triangles in $M$ with corresponding triangles in the euclidean plane, and requires that the triangle in $M$ be "no fatter" than its counterpart in the plane. More precisely, for any three points $A, B$ and $C$ in $M$, choose geodesics joining each pair, thus creating a geodesic triangle in $M$. In the plane $\mathbb{E}^{2}$, draw a corresponding triangle with vertices $A^{\prime}, B^{\prime}$ and $C^{\prime}$. The axiom requires that for any choice of points $P$ and $Q$ on different sides of the triangle in $M$ the distance $d(P, Q)$ be less than or equal to the distance in $\mathbb{E}^{2}$ between the corresponding points $P^{\prime}$ and $Q^{\prime}$. 

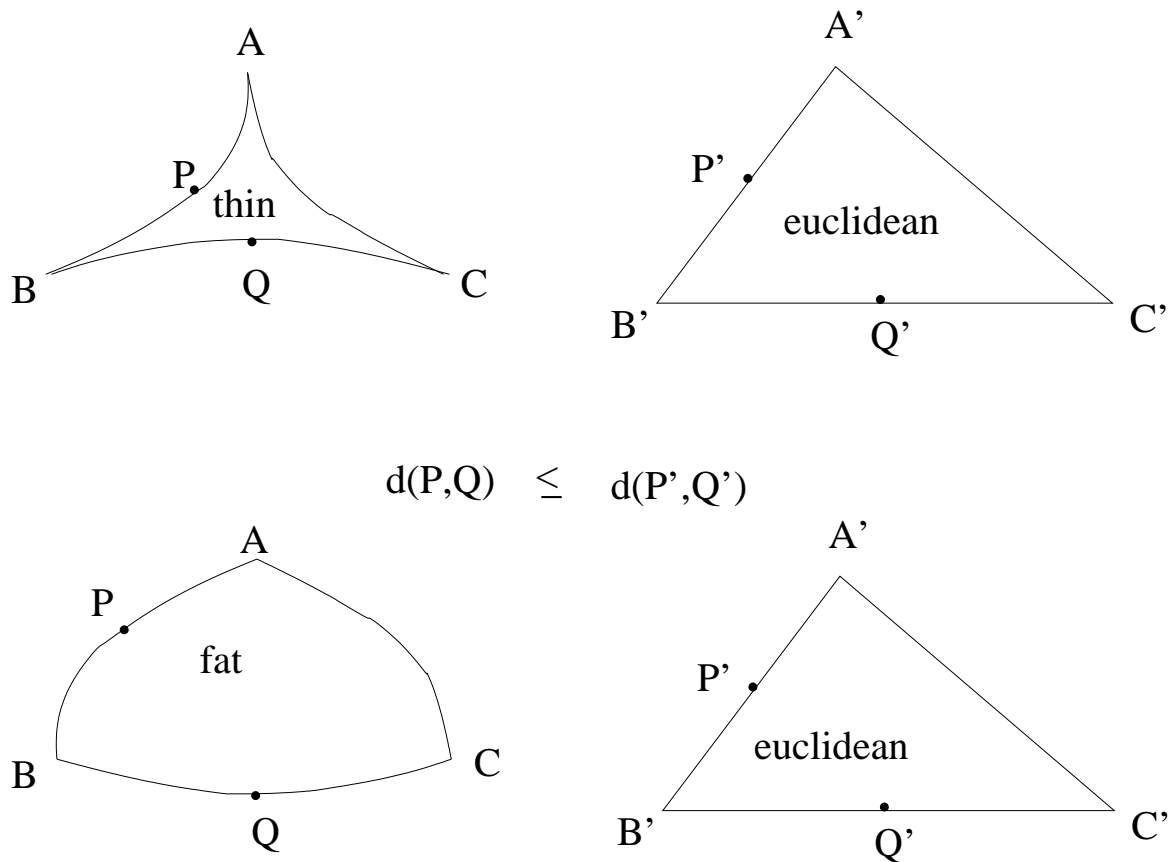

$$
\mathrm{d}(\mathrm{P}, \mathrm{Q}) \quad>\quad \mathrm{d}\left(\mathrm{P}^{\prime}, \mathrm{Q}^{\prime}\right)
$$

Remark. CAT stands for CartanAleksandrov-Toponogov.

\section{Examples of $C A T(0)$ spaces}

- Euclidean $n$-space $\mathbb{E}^{n}$.

- The hyperbolic plane $\mathbb{H}^{2}$.

- Any locally finite tree.

- Any Euclidean building.

- Any simply connected Riemannian manifold of non-positive sectional curvature

- e.g. universal cover of a compact manifold of non-positive sectional curvature.

The last of these deserves discussion. In the classical differential geometry of Riemannian manifolds there is the notion of sectional curvature at a point. This is a real number. $C A T(0)$ captures the cruder notion of "non-positive curvature everywhere", without carrying the precise information about curvature at each point. There is the more general notion of $C A T(\kappa)$ which captures the notion of "curvature $\leq \kappa$ everywhere" for a given real number $\bar{\kappa}$. The definition is similar to the $C A T(0)$ case except that a canonical space of constant curvature $\kappa$ is used for the comparison instead of the euclidean plane $\mathbb{E}^{2}$. For more on this and on all aspects of $C A T(0)$ geometry, see [1].

Remark. It follows from the Comparison Axiom that there is exactly one geodesic joining two points of $M$; for if there were two different geodesics, they would make a "fat" degenerate geodesic triangle.

Remark. $C A T(0)$ spaces are contractible. To see this, choose a point $b \in M$ as base point. The (unique) geodesic from any point $x$ to $b$ varies continuously with $x$ and thus provides a canonical contraction of all of $M$ to the singleton subspace $\{b\}$.

It is not hard to guess what it means to say that a proper geodesic metric space is "locally $C A T(0)$ ". Roughly, one asserts that the "no-fat triangles" condition holds in a neighborhood of each point. It is often important to know when such a locally $C A T(0)$ space is $C A T(0)$. Classical differential geometry suggests the answer. In the case of Riemannian manifolds the CartanHademard Theorem says that when the manifold has sectional curvature everywhere nonpositive, and is simply connected, then it is contractible. This suggests a version in the $C A T(0)$ world:

Theorem 2.1. If the proper geodesic metric space $M$ is simply connected and is locally $C A T(0)$ then $M$ is a proper $C A T(0)$ space.

For a proof of this "Cartan-Hadamard" theorem see [1].

The importance for topologists is that if a proper geodesic metric space $M_{0}$ is locally $C A T(0)$ and has fundamental group $G$ then $M_{0}$ is a $K(G, 1)$ space. This is because 
its universal cover is $C A T(0)$ and hence is contractible.

This last statement is more subtle than it looks, because $C A T(0)$ is a metric condition, so it implies a connection between the metrics on two spaces. Let $M_{0}$ be a (connected proper) locally $C A T(0)$ geodesic metric space whose universal cover, with respect to a chosen base point, is $M$. Then elementary covering space theory says that the fundamental group $G$ of $M_{0}$ acts on $M$ by homeomorphisms. In this paper it will always be understood that $G$ acts on $M$ by isometries. This implies a strong connection between the metrics on $M$ and $M_{0}$. In particular the covering projection is a local isometry, not just a local homeomorphism. The details of this are dealt with in [1] but the reader can profit by thinking out the (quite elementary) details on his/her own. Note that $M$ is a proper metric space if and only if $M_{0}$ is. In this article I will always assume that the metrics on $M_{0}$ and $M$ are compatible in this sense.

Recognition of the local $C A T(0)$ property is important. The most famous and useful recognition theorem was proved by Gromov. First, I must define the necessary terms.

(1) Consider the standard $n$-cube $I^{n}:=$ $[-1,1]^{n}$. The link of the vertex $v$ in $I^{n}$ is the convex simplex whose vertices are the mid points of the $n$ edges containing $v$.

(2) A cubical complex is a regular $C W$ complex, call it $K$, obtained from a disjoint set of standard cubes whose edges have length 1 by gluing faces of cubes together using isometries. Regularity implies that no two faces of a cube get glued together, so the cells are genuine cubes, and any two intersect in a common face.

(3) The link of a vertex $v \in K$ is the simplicial complex which is the union of the links of $v$ in each cube which contains $v$.

(4) A simplicial complex is flag if it is entirely determined by its 1-skeleton; i.e. a collection of vertices spans a simplex if and only if each pair in that collection spans an edge.

(5) A metric cubical complex is a locally finite cubical complex metrized as follows: each cube gets the standard euclidean metric; each piecewise linear path thus gets a well-defined length; the distance between any two points is defined to be the infimum of the lengths of piecewise linear paths joining those points.

Theorem 2.2. (Gromov) A metric cubical complex is locally $C A T(0)$ if and only if the link of each vertex is a flag complex.

Remark. I should add a word about the point-set topology and homotopy properties. If $M_{0}$ is a proper locally $C A T(0)$ metric space then $M_{0}$ is an absolute neighborhood retract $(A N R)$. This follows from well-established criteria found, for example, in [8]. Thus, by West's Theorem, if such a space is compact then it is homotopy equivalent to a finite $C W$ complex. Note that in general such a space might have infinite Lebesgue covering dimension. A particularly easy example of this is the Hilbert Cube, which, with the product metric, is both compact and $C A T(0)$.

In this article the $C A T(0)$ spaces of interest will always be non-compact, but they will often be universal covers of compact spaces which are locally $C A T(0)$. It need hardly be said that $C A T(0)$ spaces are locally $C A T(0)$.

Examples of non-proper and non$C A T(0)$ metric spaces

- A contractible space which somewhere has a region of positive curvature (e.g. a "bubble") does not satisfy the "nofat triangles" condition.

- Banach spaces $\ell^{p}$ satisfy the "no-fat triangles" condition if and only if $p=$ 2.

- Infinite dimensional Hilbert spaces are $C A T(0)$ but they are not proper metric spaces.

\section{$C A T(0)$ GROUPS}

The most immediate examples of what are called $C A T(0)$ groups occur as follows. One has a compact connected locally $C A T(0)$ space $M_{0}$ whose fundamental group is $G$. Then $G$ acts by isometries freely, properly discontinuously and cocompactly on the $C A T(0)$ universal cover space $M$. Such a group $G$ must be torsion free for well-known topological reasons. To allow for torsion one generalizes things slightly as follows.

A group $G$ is a $C A T(0)$ group if $G$ acts by isometries properly discontinuously and cocompactly on some proper $C A T(0)$ space. Notice that by dropping the word "free" we allow stabilizers of points to be finite subgroups of $G$ rather than just the 
trivial subgroup.

\section{Examples of $C A T(0)$ groups}

- Finitely generated abelian groups

- Finitely generated free groups

- Finitely generated Coxeter groups

- Uniform lattices in semi-simple Lie groups.

- The fundamental group of a closed Riemannian manifold of non-positive sectional curvature.

Not every finitely generated group can be a $C A T(0)$ group. Here are some necessary conditions:

- $G$ is finitely presented.

- The number of conjugacy classes of finite subgroups of $G$ is finite.

- Every solvable subgroup of $G$ is virtually abelian.

- Every abelian subgroup of $G$ is finitely generated.

For details, see Theorem III(1.1) of [1].

\section{THE BOUNDARY $\partial M$}

Fix a base point $b \in M$.

A geodesic ray in $M$ is an isometric embedding $[0, \infty) \rightarrow M$ such that 0 goes to $b$. With the compact-open topology the geodesic rays form a compact metrizable space denoted by $\partial M$. This space $\partial M$ is called the boundary of $M$ (at infinity.). This definition appears to depend on the choice of base point, but not really: another choice will give a canonically homeomorphic boundary. Or one can define the boundary in a base-pointfree way, as is done in [1].

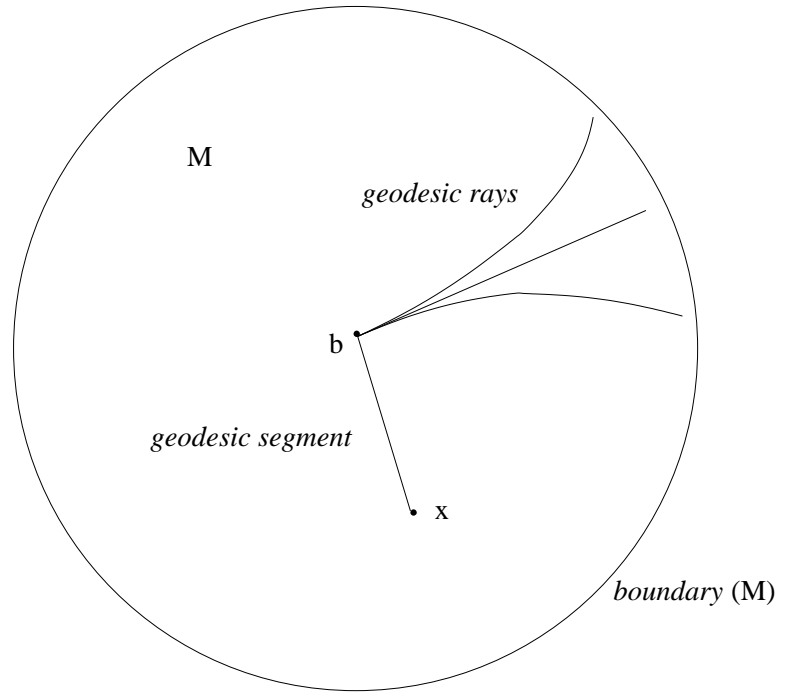

Remark. For a while people thought that perhaps the boundary might be a topological invariant of $G$. In other words, if $G$ also acts properly discontinously and cocompactly on another proper $C A T(0)$ space then the two boundaries would be homeomorphic. This turned out not to be true; counterexamples can be found in [2] and [10]. However, the two boundaries are shape equivalent, as I observed long ago in [4].

There is an important sense in which the boundary $\partial M$ compactifies $M$. For each $x \in M$ there is a geodesic $[0, d(b, x)] \rightarrow M$ from $b$ to $x$ We call this a geodesic segment just as we called the infinite version a geodesic ray. The latter have domain $[0, \infty)$. By a simple trick we can represent the geodesic segment from $b$ to $x$ also to have domain $[0, \infty)$; namely use the previous map on $[0, d(b, x)]$ and send all of $[d(b, x), \infty)$ to the point $x$. Then all the geodesic segments and all the geodesic rays (starting at $b$ ) can be discussed together as a function space $\widehat{M}$ with the compact-open topology. The ones of infinite length form $\partial M$ as before, and the ones of finite length form a copy of $M$ (which we will not distinguish from the previous $M$ ). The Arzela-Ascoli Theorem shows that $\widehat{M}$ is compact and metrizable. It is easy to see that $M$ is open and dense in $\widehat{M}$.

Here are a few examples:

- If $M=$ Euclidean $n$-space $\mathbb{E}^{n}$ then $\partial M \cong S^{n-1}$ and $\widehat{M}$ is an $n$-ball.

- If $M$ is the hyperbolic plane $\mathbb{H}^{2}$ then $\partial M \cong S^{1}$ and $\widehat{M}$ is a disk.

- If $M$ is a homogeneous locally finite tree of constant valence $>1$ then $\partial M$ is a Cantor set and $\widehat{M}$ compactifies $M$ by adding an end point to every ray.

We note that every isometry of $M$ extends to a homeomorphsism of $\widehat{M}$ which of course maps $\partial M$ to itself homeomorphically. Thus our $C A T(0)$ group acts by homeomorphisms on $\partial M$.

\section{NOTIONS OF CONNECTEDNESS FOR $\partial M$}

Every mathematician is familiar with the notion of connected component and the finer notion of path component of $\partial M$. In between these two extremes lie other forms of "component" that are less familiar. I will define two of these here.

A proper ray in $M$ is a map $\rho$ : $[0, \infty) \rightarrow M$ having the property that for every compact subset $C$ of $M$ the pre-image $\rho^{-1}(C)$ is a compact subset of $[0, \infty)$. Examples are the geodesic rays. A proper homotopy between two proper rays $\rho$ and $\sigma$ is a 
$\operatorname{map} \Sigma:[0, \infty) \times[0,1] \rightarrow M$ which is proper (i.e. pre-images of compact sets under $\Sigma$ are compact) and which agrees with $\rho$ [resp. with $\sigma]$ on $[0, \infty) \times\{0\}[$ resp. $[0, \infty) \times\{1\}]$. The given rays are properly homotopic if there exists such a map $\Sigma$.

Two points $p$ and $q$ of $\partial M$ are in the same strong homotopy component of $\partial M$ if, when considered as geodesic rays starting at $b$, they are properly homotopic rays.

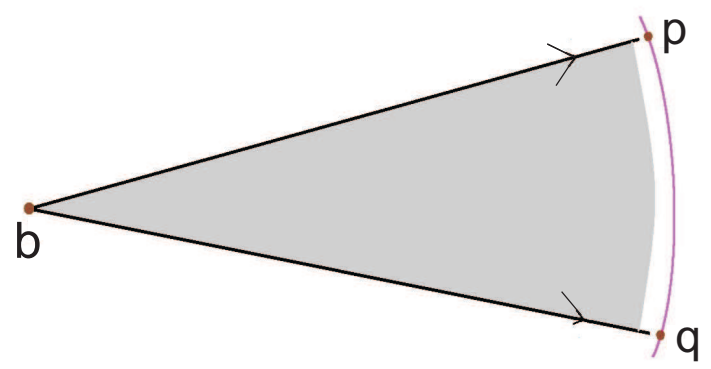

A proper homology between two proper rays $\rho$ and $\sigma$ is a proper map $T: S \times[0,1] \rightarrow$ $M$, where $S$ is an oriented 2-manifold whose boundary consists of the two rays $\rho$ and $\sigma^{1}$ The given rays are properly homologous if there exists such a map $T$.

Two points of $\partial M$ are in the same strong homology component of $\partial M$ if, when considered as geodesic rays starting at $b$, they are properly homologous rays.

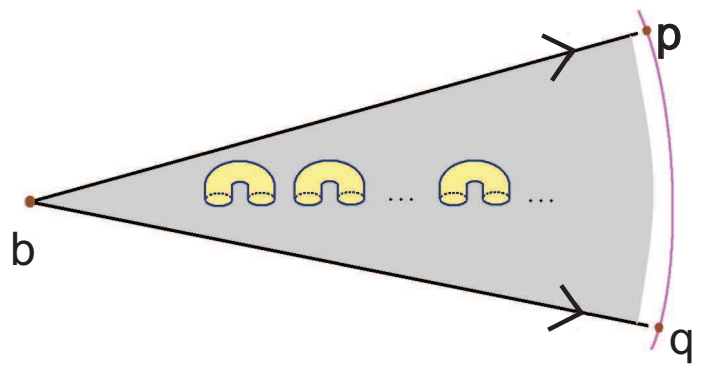

Remark. There is a related issue as to whether every proper ray in $M$ is properly homotopic (or properly homologous) to some geodesic ray. See [7] for more on this topic.

\section{GROUP-RING COHOMOLOGY OF $C A T(0)$ GROUPS}

Let $G$ be a $C A T(0)$ group acting properly and cocompactly on the proper $C A T(0)$ space $M$. The integral group-ring $\mathbb{Z} G$ becomes a $\mathbb{Z} G$-module under left translation by elements of $G$, The cohomology groups $H^{*}(G, \mathbb{Z} G)$ are derived in the usual way from any free $\mathbb{Z} G$ resolution of the trivial $\mathbb{Z} G$ module $\mathbb{Z}$. If there exists a finite $K(G, 1)$ complex $K$ for $G$ then the cellular chains in the universal cover $\widetilde{K}$ form such a resolution, and it follows that $H^{*}(G, \mathbb{Z} G)$ is functorially isomorphic to $H_{c}^{*}(\widetilde{K})$, the cohomology of $\widetilde{K}$ with compact supports. Now if $G$ acts freely on $M$ and if $M_{0}$ admits such a cellulation then the same can be said of $H_{c}^{*}(M)$ since $M$ is the universal cover of $M_{0}$. With a little more technique this statement can be shown to remain true even if the $G$-action is not free, but merely proper, and if $M_{0}$ is merely homotopy equivalent (as is the case) to a finite complex. See Sections 17.5 and 17.6 of [5] for more details. Summarizing:

Proposition 6.3. There is a functorial isomorphism between $H^{*}(G, \mathbb{Z} G)$ and $H_{c}^{*}(M)$.

A straightforward exercise in algebraic topology gives

Proposition 6.4. There is a functorial isomorphism between $H_{c}^{*}(M)$ and the Čech cohomology $\check{H}^{*-1}(\partial M)$.

These two propositions link group-ring cohomology $H^{*}(G, \mathbb{Z} G)$ with the Cech cohomology of $\partial M$ and brings us to our goal.

\section{COMPARISON OF ALGEBRAIC AND TOPOLOGICAL PROBLEMS}

The theme of this talk is that algebraic problems are sometimes equivalent to topological ones, and in unexpected ways. The particular algebra involved here is the cohomology of the group $G$ with $\mathbb{Z} G$ coefficients. I will always assume that $G$ is a $C A T(0)$ group, acting cocompactly and properly discontinuously by isometries on the proper $C A T(0)$ space $M$.

\section{Cohomology has a highest non-zero dimension:}

Theorem 7.5. (Swenson [9]) When a group acts cocompactly by isometries on a proper

\footnotetext{
${ }^{1}$ Under the orientation inherited from $S$ one of the rays is oriented towards infinity and the other is oriented away from infinity.
} 
$C A T(0)$ space $M$ then $\partial M$ has finite dimension.

A consequence for $C A T(0)$ groups $G$ is:

Corollary 7.6. $H^{n}(G, \mathbb{Z} G)=0$ for sufficiently large $n$.

Non-zero cohomology in the top dimension.

Theorem 7.7. (Geoghegan and Ontaneda [6]): When a group acts cocompactly by isometries on a proper $C A T(0)$ space $M$ and $m$ is the dimension of $\partial M$ then $H^{m+1}(G, \mathbb{Z} G) \neq 0$ (and all higher cohomology groups are zero).

A consequence for $C A T(0)$ groups $G$ is:

Corollary 7.8. The cohomological dimension of $G$ is precisely (dimension of $\partial M$ ) +1 .

Cohomology in dimension 0: The following is elementary:

Proposition 7.9. For a $C A T(0)$ group $G$ the following are equivalent;

(1) $H^{0}(G, \mathbb{Z} G)=0$;

(2) $M$ is non-compact;

(3) $G$ is infinite.

From now on I assume $M$ is noncompact and hence $G$ is infinite.

\section{Cohomology in dimension 1:}

Theorem 7.10. (Hopf) When a CAT(0) group acts cocompactly and properly on $M$ then the number of connected components of $\partial M$ is 1 or 2 or is uncountably infinite.

A consequence for $C A T(0)$ groups $G$ is:

Corollary 7.11. $H^{1}(G, \mathbb{Z} G)=0$ or $\mathbb{Z}$ or $\bigoplus_{1}^{\infty} \mathbb{Z}$

i.e. The number of "ends of $G$ " is 1 or 2 or $\infty$. The last part uses a famous theorem of Stallings.

Cohomology in dimension 2: What can be said about $H^{2}(G, \mathbb{Z} G)$ ? A theorem of Farrell (see [5] for a proof) says:

Theorem 7.12. Farell $[3] H^{2}(G, \mathbb{Z} G)$ is 0 or is isomorphic to $\mathbb{Z}$ or is an infinitely generated torsion free abelian group.

The question of whether that infinitely generated abelian group is free abelian has been open for 40 years, though it is known to have a positive answer for many classes of groups. Here is the conjecture - a strengthened form of Theorem 7.12.

Conjecture 7.13. For a $C A T(0)$ group $G$, $H^{2}(G, \mathbb{Z} G)=0$ or $\mathbb{Z}$ or $\bigoplus_{1}^{\infty} \mathbb{Z}$.

\section{THE EQUIVALENT TOPOLOGICAL CONJECTURE}

In the case of one-ended $C A T(0)$ groups Conjecture 7.13 has an equivalent (and perhaps surprising) topological statement. Recall that by Proposition 7.9 and Theorem 7.10, the assumption of oneendedness implies that $\partial M$ is non-empty and connected, and that $H^{0}(G, \mathbb{Z} G)=$ $H^{1}(G, \mathbb{Z} G)=0$

Theorem 8.14. Under these assumptions $\partial M$ has just one strong homology component if and only if $H^{2}(G, \mathbb{Z} G)$ is free abelian.

Thus we have the equivalent Conjecture

Conjecture 8.15. Under these assumptions $\partial M$ has only one strong homology component.

Remark. Indeed, there is a stronger conjecture - namely that $\partial M$ has only one strong homtopy component. This is also open and is known as the "Semistability Conjecture". Unlike the homology version, it does not have a familiar algebraic restatement. However, in almost all cases where Conjecture 8.15 has been proved, it is this stronger statement which is proved. This is normally done using fundamental group methods.

Remark. Conjecture 8.15 is in fact a conjecture in Steenrod homology, a notion closely related to shape theory.

\section{REFERENCES}

[1] Martin R. Bridson and André Haefliger. Metric spaces of non-positive curvature, volume 319 of Grundlehren der Mathematischen Wissenschaften [Fundamental Principles of Mathematical Sciences]. Springer-Verlag, Berlin,

[2] Christopher B. Croke and Bruce Kleiner. Spaces with nonpositive curvature and their ideal boundaries. Topology, 39(3):549-556, 2000.

[3] F. Thomas Farrell. The second cohomology group of $G$ with $Z_{2} G$ coefficients. Topology, 13:313-326, 1974.

[4] Ross Geoghegan. The shape of a group - connections between shape theory and the homology of groups. In Geometric and algebraic topology, volume 18 of Banach Center Publ., pages 271-280. PWN, Warsaw, 1986.

[5] Ross Geoghegan. Topological methods in group theory volume 243 of Graduate Texts in Mathematics. Springer, New York, 2008.

[6] Ross Geoghegan and Pedro Ontaneda. Boundaries of cocompact proper CAT(0) spaces. Topology, 46(2):129-137, 2007

[7] Ross Geoghegan and Eric Swenson. On semistability of CAT(0) groups. Groups, Geometry and Dynamics (to appear).

[8] Sze-tsen Hu. Theory of retracts. Wayne State University Press, Detroit, 1965.

[9] Eric L. Swenson. A cut point theorem for CAT(0) groups. J. Differential Geom., 53(2):327-358, 1999.

[10] Julia M. Wilson. A CAT(0) group with uncountably many distinct boundaries. J. Group Theory, 8(2):229238,2005 . 